\title{
Feasibility study of ultra-low-dose dedicated maxillofacial computed tomography using filter-based spectral shaping in patients with craniofacial trauma: assessment of image quality and radiation dose
}

\author{
Ji Young $\mathrm{Ha}^{1}{ }^{\wedge}$, Hye Jin Baek ${ }^{1,2}$, Kyeong Hwa Ryu ${ }^{1} \wedge$, Eun $\mathrm{Cho}^{1 \wedge}$ \\ ${ }^{1}$ Department of Radiology, Gyeongsang National University School of Medicine and Gyeongsang National University Changwon Hospital, \\ Changwon, Republic of Korea; ${ }^{2}$ Department of Radiology, Institute of Health Sciences, Gyeongsang National University School of Medicine, Jinju, \\ Republic of Korea \\ Correspondence to: Hye Jin Baek, MD, PhD. Department of Radiology, Gyeongsang National University School of Medicine and Gyeongsang National \\ University Changwon Hospital, 11 Samjeongja-ro, Seongsan-gu, Changwon 51472, Republic of Korea; Department of Radiology, Institute of Health \\ Sciences, Gyeongsang National University School of Medicine, 816-15 Jinju-daero, Jinju 52727, Republic of Korea. Email: sartre81@gmail.com.
}

Background: In the setting of multiple trauma, radiation exposure is considered a relevant issue because
patients may require repeated imaging to evaluate injuries in different body parts. Recently, spectral shaping
of the X-ray beam has been shown to be beneficial in reducing radiation exposure. We investigated the
clinical feasibility of a tin-filtered $100 \mathrm{kV}$ protocol for the diagnostic use, compared to routine dedicated
maxillofacial CT at $120 \mathrm{kVp}$ in patients with craniofacial trauma; we assessed the image quality, radiation
dose, and interobserver agreement. Methods: We retrospectively evaluated 100 consecutive patients who underwent dedicated maxillofacial CT for craniofacial trauma. Fifty patients were examined with a tin-filtered $100 \mathrm{kV}$ protocol performed using a thirdgeneration dual source CT. The other 50 patients were examined with a standard protocol on a different scanner. Two readers independently evaluated image quality subjectively and objectively, and the interobserver agreement was also assessed. CT dose index volume $\left(\mathrm{CTDI}_{\mathrm{vol}}\right)$ and dose-length product (DLP) were recorded to compare radiation exposure. A quality-control phantom was also scanned to prospectively assess the impact of tin filtration. Results: All CT scans showed diagnostic image quality for evaluating craniofacial fractures. The tinfiltered $100 \mathrm{kV}$ protocol showed sufficient-to-good image quality for diagnostic use; however, overall image quality and anatomic delineation from the tin-filtered $100 \mathrm{kV}$ protocol were significantly lower than from the standard protocol. Interobserver agreement was moderate to almost perfect $(\mathrm{k}=0.56-0.85)$. Image noises in the air, eye globe, and retrobulbar fat were comparable between the two protocols $(\mathrm{P}>0.05)$, whereas both signal-to-noise ratio and contrast-to-noise ratio in the eye globe and retrobulbar fat showed a significant difference $(\mathrm{P}<0.05)$. The tin-filtered $100 \mathrm{kV}$ protocol showed a significant reduction in radiation dose compared to the standard protocol: $\mathrm{CTDI}_{\mathrm{vol}}, 3.33$ vs. $30.5 \mathrm{mGy}(\mathrm{P}<0.001)$; and DLP, 70.70 vs. $669.43 \mathrm{mGy}^{*} \mathrm{~cm}(\mathrm{P}<0.001)$. The phantom study also demonstrated a lower radiation dose for the tin-filter $100 \mathrm{kV}$ protocol compared to the standard protocol.

Conclusions: Dedicated maxillofacial CT using spectral shaping with tin filtration can allow a significant reduction in radiation dose while maintaining sufficient diagnostic image quality, when compared to the standard protocol.

Keywords: Low dose CT; maxillofacial CT; Tin filtration; spectral shaping; trauma

Submitted Jun 26, 2020. Accepted for publication Oct 23, 2020.

doi: $10.21037 /$ qims-20-800

View this article at: http://dx.doi.org/10.21037/qims-20-800

^ ORCID: Ji Young Ha, 0000-0001-5769-3045; Kyeong Hwa Ryu, 0000-0003-1599-8881; Eun Cho, 0000-0001-7814-7430. 


\section{Introduction}

Trauma is a major cause of morbidity and mortality, and a significant proportion of patients with multiple trauma experience maxillofacial injuries $(1,2)$. The maxillofacial CT is considered the standard modality for evaluating craniofacial injuries, due to its high spatial resolution and speed $(3,4)$. However, the dedicated maxillofacial CT examination is not routinely performed for patients with multiple traumas in a real-world setting, because the maxillofacial bones are not considered the highest priority, and head or cervical spine CT imaging often includes substantial portions of the maxillofacial bones $(3,5)$. This diagnostic workflow may inadequately assess for the injuries of the maxillofacial skeleton; this causes facial deformities, resulting in cosmetic or functional problems of the corresponding anatomical units. Therefore, the dedicated maxillofacial CT has the advantage of providing a detailed evaluation of the maxillofacial anatomy and adequately aiding surgical planning for repair $(3,5)$.

However, in the setting of multiple trauma, radiation exposure is considered a relevant issue because patients may require repeated imaging to evaluate for injuries in different body parts. In particular, the maxillofacial CT (imaging from the top of the frontal sinus to the mandible) partially overlaps the scan range of the head CT (imaging from the orbitomeatal line to the vertex), therefore, an increased radiation dose is inevitable (4). Furthermore, the maxillofacial CT includes the radiosensitive optic lens, which can be a major concern for radiation exposure. Therefore, there is a clinical need to minimize the need for repetitive radiation exposure while providing detailed anatomical evaluation for patients with traumatic facial injuries.

From this perspective, various efforts have been proposed to reduce radiation exposure such as orbital bismuth shielding, the iterative reconstruction, or reduction of tube voltage and tube current (6-10). Recently, spectral shaping of the $\mathrm{X}$-ray beam using a tin (Sn) filter has been introduced as another strategy to reduce the radiation dose in dual source CT systems. The tin filter, which is added to the standard aluminum bowtie filter between the X-ray tube and the patient, absorbs low-energy photons that contribute less to the image quality, but increase the radiation exposure to patients. Therefore, the filter results in an increase in the mean photon energy of the applied radiation, and this can be less relevant in high-contrast settings (such as bone or lung imaging) (11). Several recent studies using paranasal sinus CT demonstrated that spectral shaping with a tin filter reduced the radiation dose with acceptable image quality (12-15). However, these studies mainly focused on inflammatory sinus diseases. Therefore, we hypothesized that the dedicated maxillofacial CT examination, with spectral shaping by a tin filter in the third-generation dual source CT system, can reduce the radiation dose while maintaining image quality.

In this study, we investigated the clinical feasibility of a tin-filtered $100 \mathrm{kV}$ protocol $(\mathrm{Sn} 100-\mathrm{kVp})$ in comparison with a routine dedicated maxillofacial CT at $120 \mathrm{kVp}$ (routine $120-\mathrm{kVp}$ ) in patients with craniofacial trauma; we accomplished this by assessing the image quality, radiation dose, and interobserver agreement.

\section{Methods}

\section{Patient population}

Our Institutional Review Board of Gyeongsang National University Changwon Hospital (No.: GNUCH 2019-11018) approved this study, and the requirement for informed consent was waived due to the study's retrospective nature. All data were obtained as part of the routine clinical examination of each patient, and the patient's records and information were anonymized and de-identified prior to analysis.

From January 2019 to July 2019, we retrospectively reviewed patients who visited the emergency room and underwent maxillofacial CT for suspected traumatic fracture of facial bones. Fifty consecutive patients (34 males, 16 females; mean age, $52.1 \pm 16.3$ years; age range, 23-83 years) who underwent a low-dose maxillofacial CT with tin filtration between April 2019 and July 2019 were included in the study group. This study group was compared to a control group of consecutive patients $(\mathrm{n}=50$, 32 males, 18 females; mean age, $52.6 \pm 15.8$ years; age range, 22-84 years) who underwent a standard-dose maxillofacial CT on a different scanner between January 2019 to May 2019. In all patients, no study had to be repeated.

\section{Imaging scan parameters}

The examinations in the study group were performed on third-generation dual source CT scanner (Siemens SOMATOM Force; Siemens Healthcare, Forchheim, Germany). The following acquisition parameters were applied: $100 \mathrm{kVp}$ with tin $(\mathrm{Sn})$ pre-filtration, activated 
automated tube current modulation (CARE Dose 4D, Siemens); iterative reconstruction (Advanced Modeled Iterative Reconstruction, level 2); collimation, $192 \mathrm{~mm}$ $\times 0.6 \mathrm{~mm}$; pitch factor, 0.8 ; rotation time, 1.0 seconds; field-of-view (FOV), $200 \mathrm{~mm}$; slice thickness, $2 \mathrm{~mm}$; slice increment, $2 \mathrm{~mm}$; and exposure time, 3.28 seconds. Lateral topography in the study group was obtained with the following acquisition parameters: $\mathrm{Sn} 100-\mathrm{kVp}$ and $48 \mathrm{~mA}$.

The examinations in the control group were performed on a dual-layer detector CT scanner (IQon Spectral CT, Philips Healthcare, Best, The Netherlands). The following acquisition parameters were applied: $120 \mathrm{kVp} ; 176 \mathrm{mAs}$; iterative reconstruction (iDose, level 2); collimation, $64 \mathrm{~mm}$ $\times 0.625 \mathrm{~mm}$; pitch factor, 0.39 ; rotation time, 0.4 seconds; FOV, $200 \mathrm{~mm}$; slice thickness, $2 \mathrm{~mm}$; slice increment, $2 \mathrm{~mm}$; and exposure time, 5.0 seconds. Lateral topography in the control group was obtained with the following acquisition parameters; $120 \mathrm{kVp}$ and $30 \mathrm{~mA}$.

All patients in both groups were scanned in the supine position with the head slightly reclined, in order to obtain a parallel alignment of the upper jaw to the gantry, to minimize artifacts from dental prostheses. The scan range included the roof of the frontal sinuses to the inferior wall of the mandible.

\section{Subjective assessment of image quality}

All data sets were anonymized and randomized. Two boardcertified radiologists (readers $\mathrm{A}$ and $\mathrm{B}$, with 7 years of experience in neuroradiology and 6 years of experience in pediatric radiology, respectively) were blinded to patient's information and CT protocols. They reviewed all images using the picture archiving and communication system (PACS). Overall image quality and the differentiability of common anatomic landmarks were assessed using a 5-point Likert scale, as follows: 1, non-diagnostic; 2, diagnostic uncertainty with substantial image noise and artifacts; 3 , diagnostic, average image quality with a correct diagnosis being highly likely; 4, diagnostic, good image quality, enabling a confident diagnosis with mild image noise and artifacts; and 5, diagnostic, excellent image quality with absent or subtle noise and artifacts, resulting in the best diagnostic value. During the analysis period, the readers also evaluated bony structures (the nasal septum, ostiomeatal complex, nasal turbinates, ethmoid air cells, orbital wall, mastoid cells, and carotid canal) and soft tissues (the eye globe, extraocular muscles, orbital fat, and optic nerve).

\section{Objective assessment of image quality}

To obtain and compare objective parameters between the two data sets, noise $(\mathrm{N})$, signal-to-noise ratio (SNR), and contrast-to-noise ratio (CNR) were calculated by one reader using circular regions of interest (ROIs). ROIs were placed in the eye globes, retrobulbar fat, and air anterior to the face at the level of the maxillary sinus. ROIs with at least an area of $2 \mathrm{~mm}^{2}$ were measured to obtain an attenuation value (AV). $\mathrm{N}$ was considered the standard deviation of the measurement in each of the ROIs. To account for anatomic differences between patients, the ROIs were chosen as large and as similar as possible while carefully avoiding image artifacts and adjacent structures, in order to prevent partial volume effects. To minimize intra-reader variability, each of the measurements were performed on both sides and repeated twice; the mean of these measurements was subsequently calculated. For example:

$$
\begin{aligned}
& \mathrm{AV}_{\text {eye globe }}=\left[\frac{\left(A V_{1 \_ \text {right }}+A V_{1 \_ \text {left }}\right)}{2}+\frac{\left(A V_{2_{-} \text {right }}+A V_{2 \_ \text {left }}\right)}{2}\right] / 2 \\
& \mathrm{~N}_{\text {air }}=\left[\frac{\left(N_{1 \_ \text {right }}+N_{1 \_ \text {left }}\right)}{2}+\frac{\left(N_{2 \_ \text {right }}+N_{2 \_ \text {left }}\right)}{2}\right] / 2
\end{aligned}
$$

The SNRs of the eye globe and retrobulbar fat were determined according to the following equation: SNR $=\mathrm{AV} / \mathrm{N}_{\mathrm{air}}$. CNRs were calculated by division of the attenuation differences between the eye globes and air as well as retrobulbar fat and eye globes with $\mathrm{N}$ according to the equation:

$$
\begin{aligned}
& \mathrm{CNR}_{\text {eye globe/air }}=\frac{\left(A V_{\text {eye globe }}-A V_{\text {air }}\right)}{\text { average Nof eye globes, retrobulbar fat, and air }} \\
& \mathrm{CNR}_{\text {eye globe/retrobulbar fat }}=\frac{\left(A V_{\text {eye globe }}-A V_{\text {retrobulbar fat }}\right)}{\text { average N of eye globes, retrobulbar fat, and air }}
\end{aligned}
$$

\section{Estimation of radiation exposure}

The estimation of the radiation dose associated with CT examination including lateral topography was based on the CT dose index volume $\left(\mathrm{CTDI}_{\mathrm{vol}}\right)$ and dose-length product (DLP) provided by the scanner. The effective radiation dose of a CT examination was calculated using the following formula, in accordance with the International Commission on Radiological Protection Publication 103 (ICRP 103) (16):

$$
\mathrm{DLP}[\mathrm{mGy} \times \mathrm{cm}] \times 0.0019\left[\mathrm{mSv} \times \mathrm{mGy}^{-1} \times \mathrm{cm}^{-1}\right]
$$


A
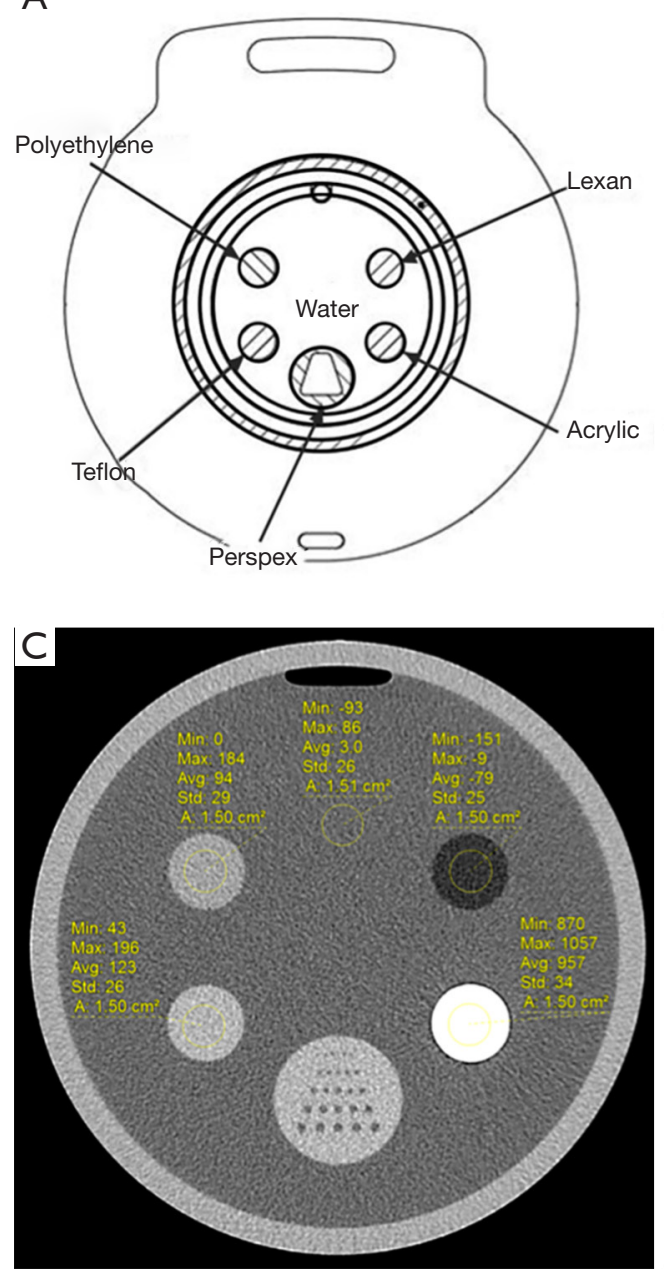

B
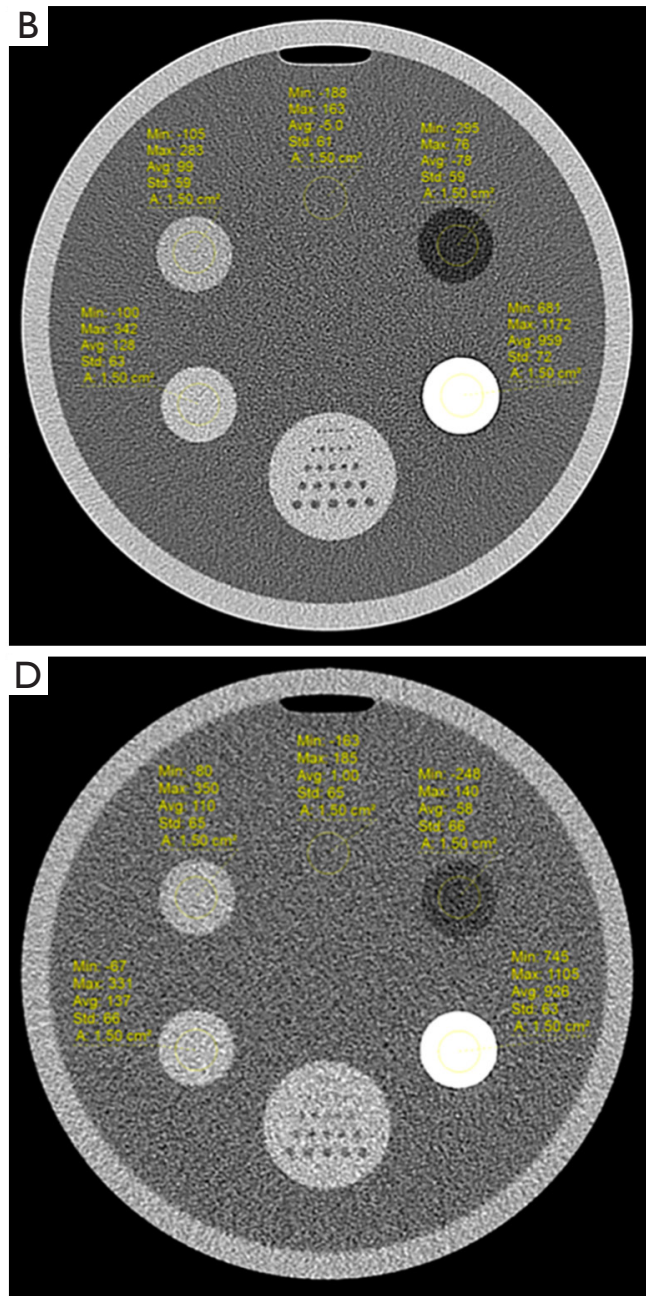

Figure 1 Representative CT images from phantom study on the two different scanners. (A) Schematic diagram of CT quality control phantom shows five different materials: water, polyethylene, Teflon, acrylic, and Lexan. (B,C,D) Image noise and attenuation value measurement in five circular regions of interest (ROIs) in different materials. (B) Routine 120-kVp protocol using the dual-layered spectral CT; $120 \mathrm{kVp}$. (C) Routine 100-kVp protocol using the third-generation dual energy CT; $100 \mathrm{kVp}$ without tin filtration, and (D) Sn100-kVp protocol using the third-generation dual energy CT; $100 \mathrm{kVp}$ with tin pre-filtration.

\section{Phantom study}

In the final phase of this study, we also scanned a phantom to prospectively assess the impact of tin filtration by comparing the performance such as basic image quality properties of the $\mathrm{Sn} 100-\mathrm{kVp}$ protocol, $100-\mathrm{kVp}$ without a tin filter, and routine $120-\mathrm{kVp}$ protocol on the two different CT scanners. Commercially available quality-control phantom (Philips Healthsystems, Cleveland, Ohio, USA) was used. The phantom consisted of five independent parts that can measure the CT number of different materials (i.e., water, polyethylene, Teflon, acrylic, and Lexan), illustrated in Figure 1A. In addition, the same modeled iterative reconstructions were applied to the phantom scan. Finally, the three different phantom scans were obtained for review; first, $\mathrm{Sn} 100-\mathrm{kVp}$ protocol using third-generation dual source CT scanner at $200 \mathrm{mAs}$; second, $100-\mathrm{kVp}$ protocol without a tin filtration using third-generation dual source CT scanner at $200 \mathrm{mAs}$; and third, routine $120-\mathrm{kVp}$ protocol using a dual-layer detector CT scanner at $176 \mathrm{mAs}$. The $\mathrm{AV}$ and $\mathrm{N}$ of the five different materials were obtained using an approximately $1.5 \mathrm{~cm}^{2}$ ROI (Figure $1 B, C, D)$. SNRs of water and polyethylene were calculated according to the following equation: $\mathrm{SNR}=\mathrm{AV} / \mathrm{N}_{\text {mean }}$. Dose 
Table 1 Subjective image quality for all evaluated anatomic landmarks

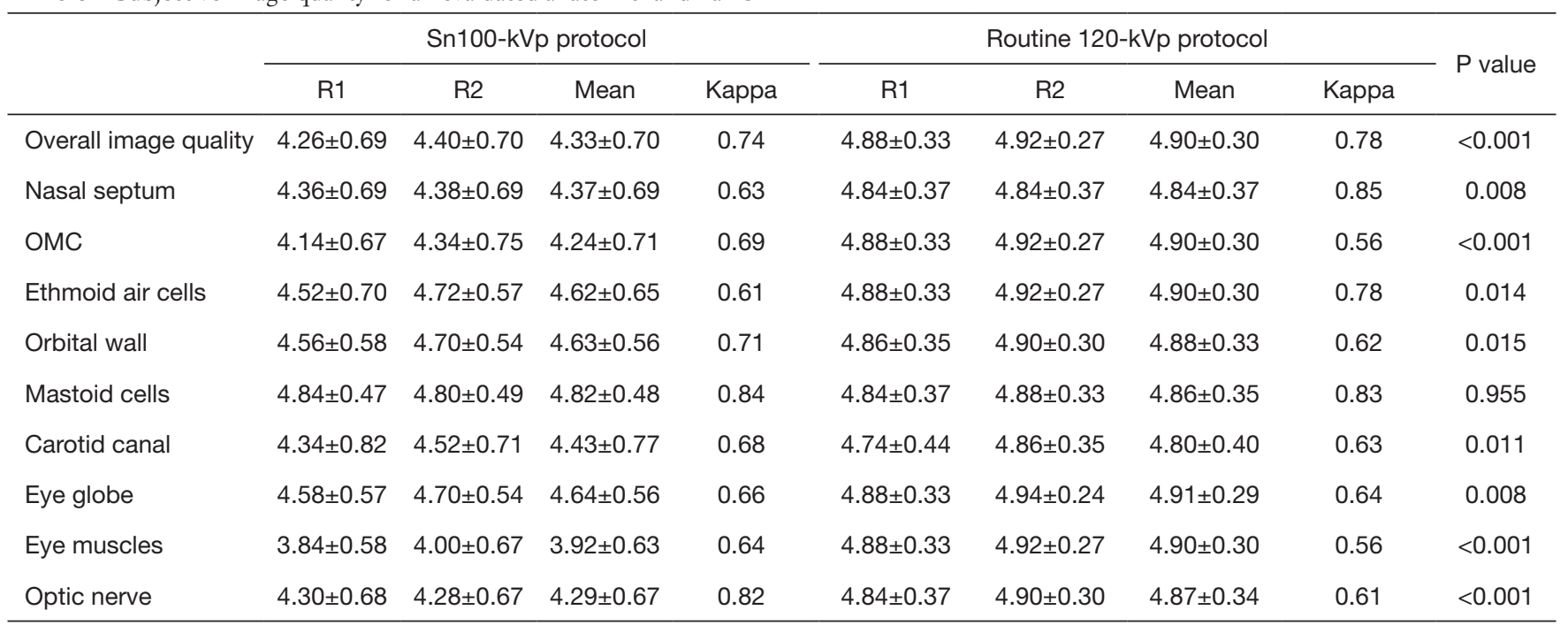

Values are mean \pm SD; OMC, ostiomeatal complex.

data of the phantom scans, including CTDI $_{\mathrm{vol}}$ and DLP, were extracted from the scanners.

\section{Statistical analyses}

The normality of the continuous variables was tested using the Kolmogorov-Smirnov test. The non-normally distributed continuous variables (AV, N, SNR, CNR, $\mathrm{CTDI}_{\mathrm{vol}}, \mathrm{DLP}$, and effective radiation dose) and the ordinal variable (subjective image quality) were analyzed using the Mann-Whitney $U$ test and are presented as medians \pm interquartile ranges. The Mann-Whitney $U$ test was performed for the comparison of subjective and objective image quality, and for the comparison of radiation exposure between both groups. The interobserver agreement for the subjective image quality between the two readers was assessed using Cohen's weighted kappa $(\kappa)$ test; the degree of agreement ( $\kappa$ value) was categorized as follows: $<0$, poor; $0-0.20$, slight agreement; $0.21-0.40$, fair agreement; 0.41-0.60, moderate agreement; $0.61-0.80$, substantial agreement; and 0.81-1, almost perfect agreement (17).

All statistical analyses were performed with statistical software (SPSS, version 24.0; IBM Corp., Armonk, NY, USA). A two-sided $\mathrm{P}$ value of less than 0.05 was considered to indicate a statistically significant difference.

\section{Results}

In the study group, 22/50 (44\%) patients had a maxillofacial fracture. In the control group, 18/50 (36\%) patients had a facial bone fracture. The two readers did not have any difficulty in detecting facial bone fractures during the analysis of all 100 image sets in the two different groups.

\section{Subjective image quality analyses}

The image quality scores and the corresponding interobserver agreement for all evaluated structures are shown in Table 1. The scores showed moderate to almost perfect interobserver agreement, ranging from 0.56 to 0.85 for all items.

Although the image quality of the study group following the $\mathrm{Sn} 100-\mathrm{kV}$ p protocol was significantly lower than the corresponding values for the routine protocol, the Sn100$\mathrm{kVp}$ protocol showed sufficient-to-good image quality with a score greater than 3 points. For the $\mathrm{Sn} 100-\mathrm{kV}$ p protocol, anatomic demarcation of the mastoid cells demonstrated good-to-excellent results in assessments without significant difference compared to the routine $120-\mathrm{kVp}$ protocol. Representative image examples of both CT protocols are shown in Figures 2,3.

\section{Objective image quality analyses}

A summary of all calculated objective image quality analyses is shown in Table 2. The AVs in the air, eye globe, and retrobulbar fat were significantly different between the Sn $100-k V p$ and routine $120-k V p$ protocols $(\mathrm{P}<0.001)$. 

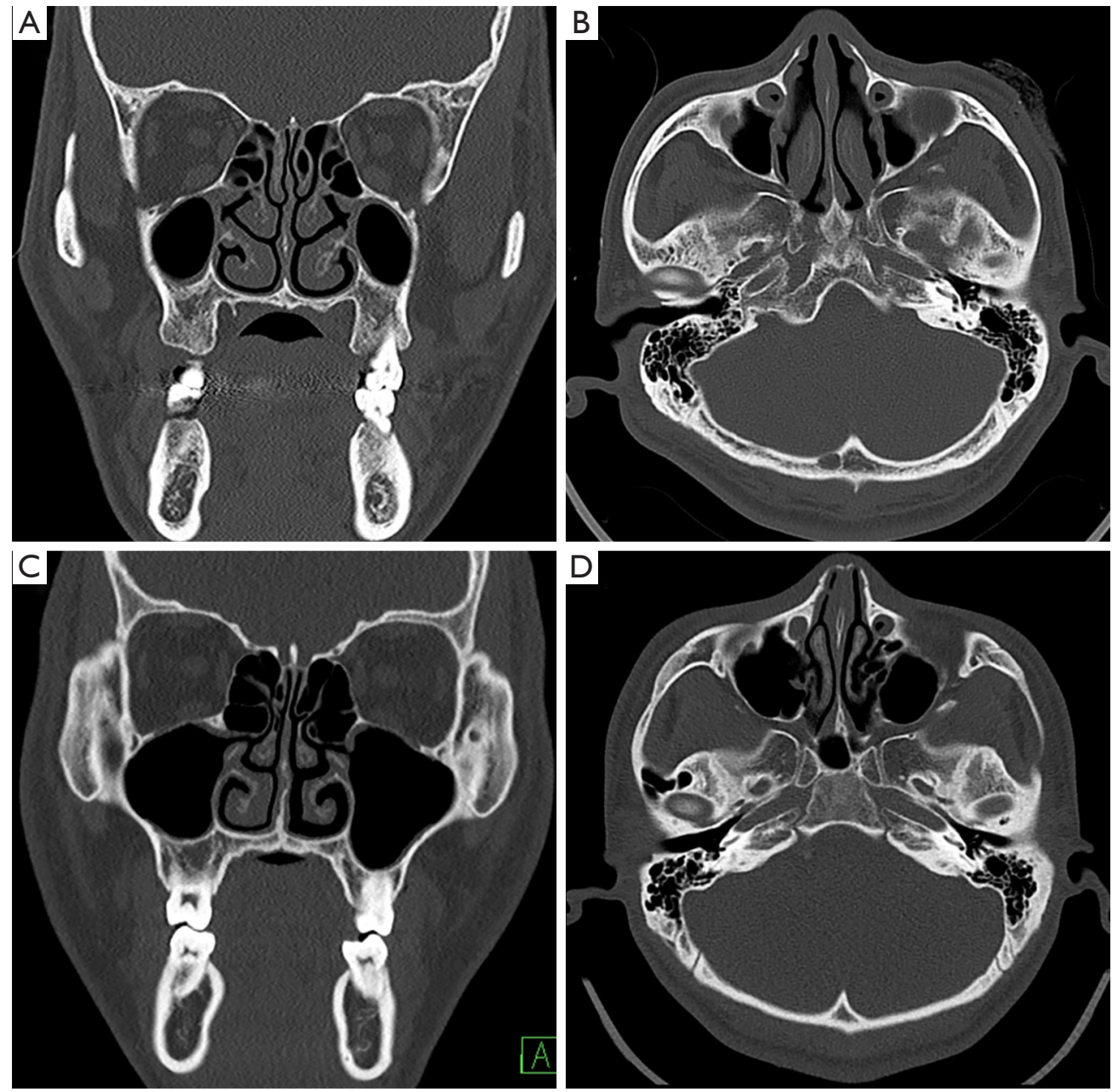

Figure 2 Representative images of the control group following the routine $120-\mathrm{kV}$ p protocol (upper row, A,B) and of the study group following the Sn100-kVp protocol (lower row, C,D). Bony structures are clearly defined in both groups.

However, the $\mathrm{N}$ in the air, eye globe, and retrobulbar fat was not significantly different between the two groups $(\mathrm{P}>0.05)$. The SNR for both the eye globe and retrobulbar fat showed a significant difference between the two groups $(\mathrm{P}<0.001)$. In addition, the CNR for the eye globe and air demonstrated a marginal difference between the two groups $(\mathrm{P}=0.054)$, whereas the $\mathrm{CNR}$ for the eye globe and retrobulbar fat showed a significant difference between the two groups $(\mathrm{P}<0.001)$.

\section{Radiation exposure analyses}

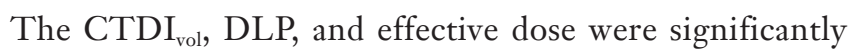
reduced in the study group with $\mathrm{Sn} 100-\mathrm{kVp}$ scans compared to the control group undergoing routine $120-\mathrm{kVp}$ scans
$(\mathrm{P}<0.001)$; all of the median radiation doses for $\mathrm{Sn} 100-\mathrm{kVp}$ protocol were reduced by more than $50 \%$ compared to the doses for the routine protocol (Table 3). In addition, $\mathrm{CTDI}_{\mathrm{vol}}$ and DLP of the lateral topography were also significantly reduced in the study group following the $\mathrm{Sn} 100-\mathrm{kVp}$ protocol $(\mathrm{P}<0.001)$.

\section{Phantom study}

The AVs and $\mathrm{N}$ of five different materials and SNR of water and polyethylene are shown in Table 4. The CTDI ${ }_{\mathrm{vol}}$ and DLP of Sn100-kVp protocol were reduced by more than $90 \%$, compared to routine $100-\mathrm{kVp}$ protocol with the same CT scanner: CTDI $\mathrm{v}_{\mathrm{vol}}, 1.66$ vs. $17.66 \mathrm{mGy}$, respectively; and DLP, 44.0 vs. $463.5 \mathrm{mGy}^{*} \mathrm{~cm}$, respectively. 

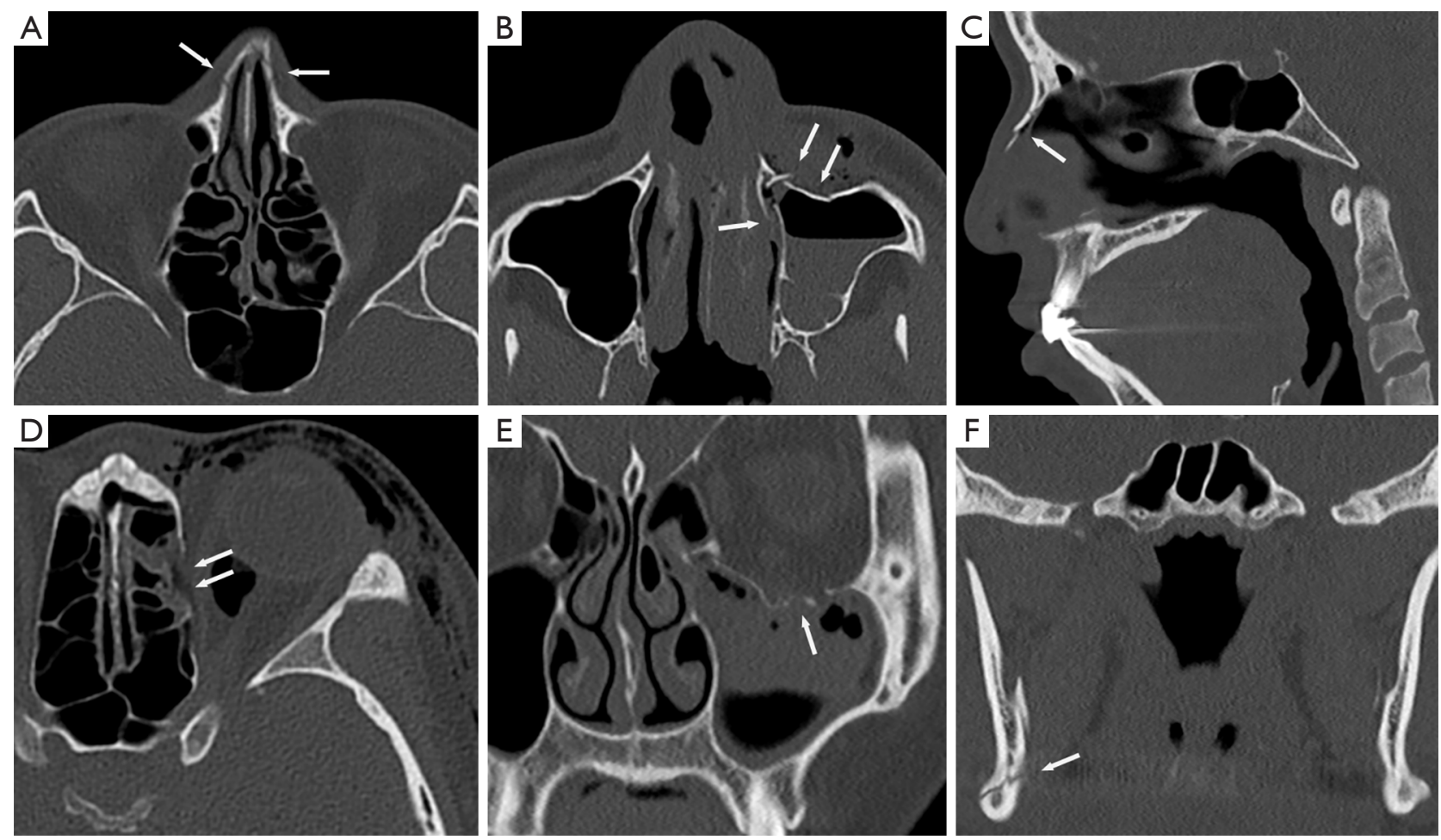

Figure 3 Image obtained from the $\mathrm{Sn} 100-\mathrm{kV}$ p protocol in six patients with fractures in different locations. (A) Axial image showing fractures in bilateral nasal bones and the right nasomaxillary junction (arrows). (B) Axial image showing fractures of the anterior and medial walls of the left maxillary sinus (arrows). (C) Sagittal image showing a depressed fracture in the nasal tip (arrow). (D) Axial image showing an acute blow-out fracture of the left medial orbital wall (arrows). Note the soft tissue swelling and emphysema in the left periorbital soft tissue and orbit. (E) Coronal image showing a fracture of the left inferior orbital wall involving the ipsilateral infraorbital canal. There is focal downward herniation of infraorbital fat through the fracture site (arrow). (F) Coronal image showing an oblique fracture line in the right mandibular angle (arrow).

Table 2 Results of objective image quality

\begin{tabular}{|c|c|c|c|}
\hline & Sn100-kVp & Routine $120-k V p$ & $P$ value \\
\hline$A V_{\text {eye globe }}$ & $4.12 \pm 4.32$ & $8.98 \pm 4.10$ & $<0.001$ \\
\hline$A V_{\text {retrobulbar fat }}$ & $-84.58 \pm 6.92$ & $-95.06 \pm 3.81$ & $<0.001$ \\
\hline $\mathrm{N}_{\text {air }}$ & $23.68 \pm 4.14$ & $23.16 \pm 3.71$ & 0.475 \\
\hline $\mathrm{N}_{\text {retrobulbar fat }}$ & $44.90 \pm 6.00$ & $44.82 \pm 4.24$ & 0.788 \\
\hline SNR eye globe & $0.18 \pm 0.20$ & $0.37 \pm 0.18$ & $<0.001$ \\
\hline$S N R_{\text {retrobulbar fat }}$ & $-3.68 \pm 0.72$ & $-4.21 \pm 0.74$ & 0.001 \\
\hline $\mathrm{CNR}_{\text {eye globe/air }}$ & $26.60 \pm 2.72$ & $27.43 \pm 2.22$ & 0.054 \\
\hline
\end{tabular}

$\mathrm{AV}$, attenuation values [HU]; CNR, contrast-to-noise ratio; $\mathrm{N}$, image noise [HU]; SNR, signal-to-noise ratio. 
Table 3 A summary of radiation exposure from the two different CT protocols

\begin{tabular}{|c|c|c|c|c|}
\hline & Metric & Sn100-kVp & Routine $120-k V p$ & $P$ value \\
\hline Topography & DLP & $0.83 \pm 0.71$ & $2.47 \pm 0.48$ & $<0.001$ \\
\hline \multirow[t]{2}{*}{ Maxillofacial CT } & $\mathrm{CTDI}_{\mathrm{vol}}$ & $3.33 \pm 1.01$ & 30.5 & $<0.001$ \\
\hline & DLP & $70.70 \pm 23.13$ & $669.43 \pm 64.09$ & $<0.001$ \\
\hline
\end{tabular}

$\mathrm{CTDI}_{\mathrm{vol}}, \mathrm{CT}$ dose index volume [mGy]; DLP, dose-length product [mGy* $\left.{ }^{*} \mathrm{~cm}\right]$; effective dose [mSv].

Table 4 Phantom study

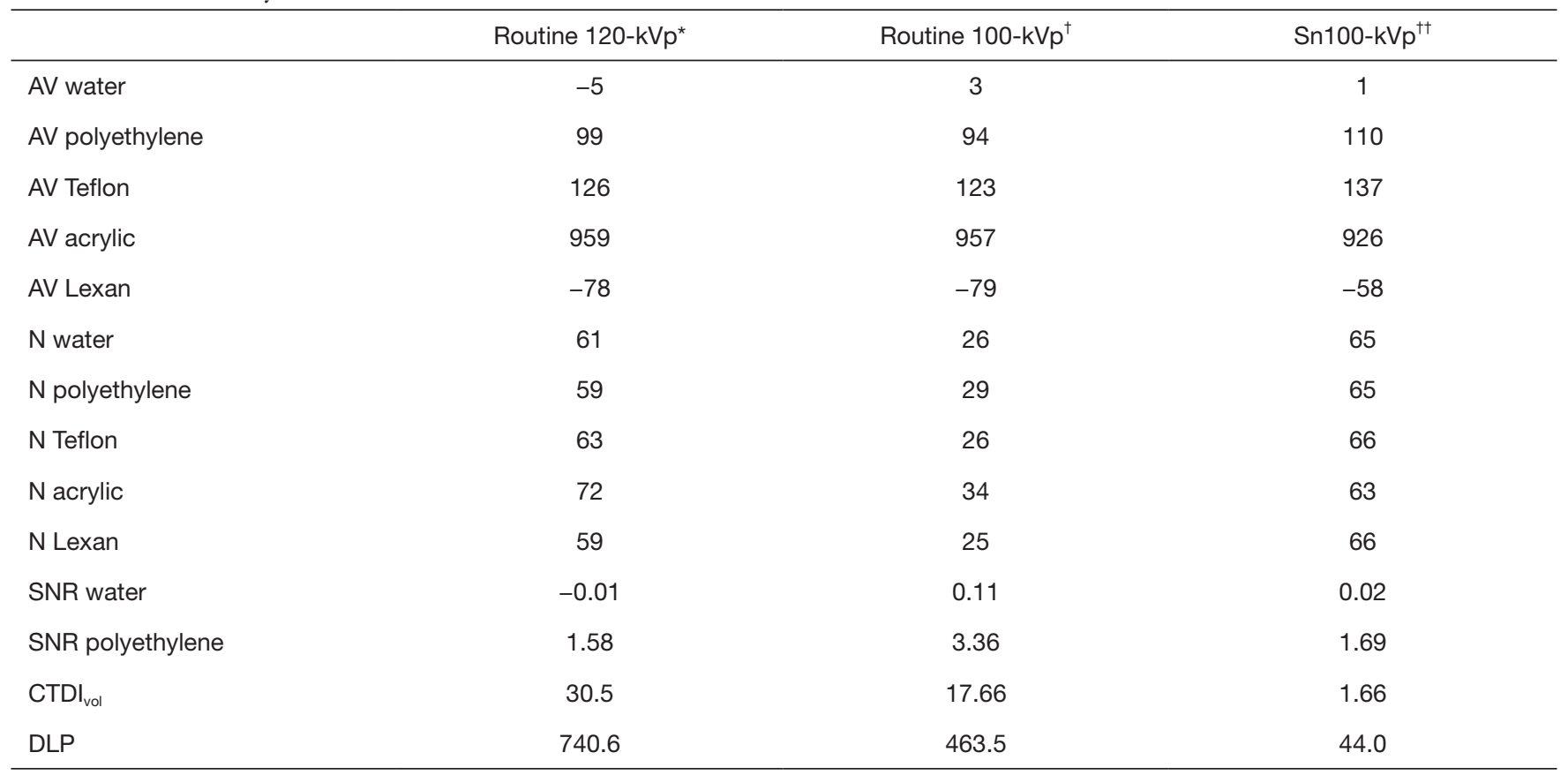

*, Philips scanner, $120 \mathrm{kVp} ;{ }^{\dagger}$, Siemens scanner, $100 \mathrm{kVp} ;{ }^{\dagger \dagger}$, Siemens scanner, $100 \mathrm{kVp}$ with tin prefiltration. AV, attenuation values [HU]; $\mathrm{CTDI}_{\mathrm{vo}}$, CT dose index volume [mGy]; DLP, dose-length product [mGy* ${ }^{*} \mathrm{~cm}$; $\mathrm{N}$, image noise [HU]; SNR, signal-to-noise ratio.

\section{Discussion}

In the current study, we assessed the radiation dose, and qualitative and quantitative diagnostic image quality of the Sn100-kVp maxillofacial CT protocol. We found that this protocol could allow for a significant reduction (more than $50 \%$ in $\mathrm{CTDI}_{\mathrm{vol}}$ and DLP) of radiation dose in patients with craniofacial trauma. In addition, the results of our study demonstrate that this ultra-low-dose CT protocol with tin filtration has the sufficient diagnostic image quality for evaluating the maxillofacial structures, supporting its clinical feasibility.

Tin filtration is an additional way of reducing the radiation dose in dual source CT systems (DSCT). This pre-filtration technique, using a tin filter in the CT tube, causes spectral shaping of the polychromatic $\mathrm{X}$-ray beam by absorbing the low levels of $\mathrm{X}$-ray energy; this contributes little to image quality, but increases the radiation dose to patients (18-20). The additional built-in tin filter with a thickness of $0.6 \mathrm{~mm}$, which removes many of lower energy photons, leads to a mean photon energy of $78.7 \mathrm{keV}$ at a fixed tube voltage of $100 \mathrm{kVp}$. This is significantly higher than the mean photon energy of $66.4 \mathrm{keV}$ with the standard $100 \mathrm{kVp}$ protocol $(11,20)$. Hence, a tin filter increases the mean photon energy level while decreasing the radiation 
dose exposure to patients, resulting in a higher dose efficiency during CT examination.

In the literature, the benefit of the tin filtration technique was initially evaluated in non-contrast chest DSCT $(11,21)$, and subsequent studies have been performed using various body parts. In abdominal imaging, several studies have demonstrated that a CT protocol with a tin filter significantly improves the material differentiation of urinary stones compared to routine CT protocol (22-24). Since the third-generation of DSCT was introduced in 2014, a tin filter can be implemented even with single energy acquisition (25). Subsequent CT studies using a single-energy mode with a tin filter $(\mathrm{Sn} 100-\mathrm{kVp})$ in adult and pediatric patients have shown that the Sn100-kVp protocol significantly reduces the radiation dose, while maintaining diagnostic image quality when compared to the conventional CT protocol $(20,26)$. In addition, the recent CT studies of the paranasal sinus and temporal bone using a tin filter (Sn100-kVp and $\mathrm{Sn} 150-\mathrm{kVp}$, respectively) also demonstrated a reduction in delivered radiation doses to patients while preserving image quality (11-14,27).

However, the previous CT studies using a tin filter in head and neck imaging mainly focused on the evaluation of inflammatory disease (12-15). Thus, the current study was aimed to investigate the clinical feasibility of dedicated maxillofacial CT examination using a single-energy mode and a tin filter ( $\mathrm{Sn} 100-\mathrm{kVp}$ protocol) specifically in craniofacial trauma. Despite the fact that maxillofacial CT is not frequently performed in the clinical practice, due to partial overlap of the scan range with head or cervical spine CT scans and the relatively lower priority given to this anatomical region, inadequate assessment of the maxillofacial bony injuries can cause subsequent cosmetic or functional problems.

In the current study, the results demonstrated similar to those of previous CT studies using a spectral shaping with a tin filter (11-15,21-27). The maxillofacial CT following the $\mathrm{Sn} 100-\mathrm{kVp}$ protocol revealed acceptable image quality for the anatomic demarcation of bony structures, suitable for diagnostic use, and allowed a significant reduction in the radiation dose given to patients. In addition, the ultralow-dose CT with the $\mathrm{Sn} 100-\mathrm{kVp}$ protocol was useful to detect cortical discontinuity of acute maxillofacial fractures. However, the image quality of the $\mathrm{Sn} 100-\mathrm{kVp}$ protocol was significantly inferior to that of the routine $120-\mathrm{kVp}$ protocol in the mastoid bone. In addition, there was a limitation in the ability of the $\mathrm{Sn} 100-\mathrm{kVp}$ protocol to evaluate fine osseous structures, such as the ear ossicles; however, it was not our intention to study this, as it is well-known that high-resolution CT images are better at visualizing the complex and detailed bony structures in the temporal bone.

Regarding the SNR, our findings demonstrated that the SNR with the Sn100-kVp protocol was significantly different from that with the routine $120-\mathrm{kVp}$ protocol. These findings are consistent with the results of the previous studies that used a low-dose CT protocol with tin filtration for paranasal sinus imaging $(13,14)$. These findings may be influenced by the difference in the AVs of water and fat, because the $\mathrm{N}$ of air, eye globe, and retrobulbar fat were not significantly different between the two protocols. Although the reason for the AV differences among the tissues between the two protocols is unclear, it is possible that the inherent characteristics of each tissue may have an impact, considering the fact that the $\mathrm{AV}$ in $\mathrm{CT}$ imaging is influenced by $\mathrm{kVp}$ or $\mathrm{keV}$, depending on the individual tissues. In addition, the directionality of the AV differences was actually consistent in this study, because the expected trend with a hardened beam would be that the $\mathrm{AV}$ differences between water and other materials would be smaller. However, further studies are required to explain this observation.

In the current study, the radiation dose was significantly lower with the $\mathrm{Sn} 100-\mathrm{kVp}$ protocol than with the routine $120-\mathrm{kVp}$ protocol. In contrast to the previous CT studies with tin filter-based spectral shaping (11-15,21-27), we assessed the radiation dose from topography in each CT examination, and to the best of our knowledge, this study is the first attempt to evaluate the radiation dose of topography. Generally, the radiation dose of topography is thought to be a negligible quantity compared to that of the CT scan itself (topography vs. CT scan; 0.83-2.47 vs. 70.7$669.43 \mathrm{mGy}^{*} \mathrm{~cm}$, respectively). Therefore, radiologists and other clinicians do not pay much attention to topography. In the present study, we found that the DLP of topography with the $\mathrm{Sn} 100-\mathrm{kV}$ p protocol was significantly reduced compared to that of standard topography. We believe that this result is meaningful and may represent an additional way to reduce the total dose of radiation exposure to patients during CT examination.

Our study has some limitations that should be considered when interpreting the findings. First, there was unavoidable selection bias because the data from all patients were evaluated retrospectively. Second, we could not obtain both $\mathrm{Sn} 100-\mathrm{kVp}$ and routine $120-\mathrm{kVp}$ CT images for the included patients, and we used two different $\mathrm{CT}$ scanners that used different protocols ( $\mathrm{kVp}$ and $\mathrm{mA})$. 
Studies with these designs should be approached carefully because of the ethical implications of repeated radiation exposure associated with prospective concurrent imaging of patients using different CT protocols. To overcome this limitation, we additionally conducted a phantom study by assessing basic image quality properties of the two different scanners and effect of the tin filtration. It may be helpful to understand the source of differences observed in the patient data because it is inevitable to control the confounding factors related to the use of two different scanners. Lastly, spectral shaping using a tin filter is not available in the vast majority of CT scanners presently.

\section{Conclusions}

Dedicated maxillofacial CT using Sn100-kVp significantly reduces the radiation dose while maintaining image quality for diagnostic use in patients with craniofacial trauma. Therefore, this ultra-low-dose CT protocol could be useful to evaluate maxillofacial injuries by overcoming radiation hazards from repetitive CT examinations in the acute trauma setting. Further CT studies using tin filtration are required to support our results in order to consider the changes in the $\mathrm{AV}$ among different tissues and a balance between contrast and noise related to dose reduction.

\section{Acknowledgments}

Funding: None.

\section{Footnote}

Conflicts of Interest: All authors have completed the ICMJE uniform disclosure form (available at http://dx.doi. org/10.21037/qims-20-800). The authors have no conflicts of interest to declare.

Ethical Statement: The study conformed to the provisions of the Declaration of Helsinki as revised in 2013. The study was approved by the Institutional Review Board of Gyeongsang National University Changwon Hospital (No.: GNUCH 2019-11-018) approved this study, and the requirement for informed consent was waived due to the study's retrospective nature.

Open Access Statement: This is an Open Access article distributed in accordance with the Creative Commons Attribution-NonCommercial-NoDerivs 4.0 International
License (CC BY-NC-ND 4.0), which permits the noncommercial replication and distribution of the article with the strict proviso that no changes or edits are made and the original work is properly cited (including links to both the formal publication through the relevant DOI and the license). See: https://creativecommons.org/licenses/by-nc-nd/4.0/.

\section{References}

1. Cannell H, Paterson A, Loukota R. Maxillofacial injuries in multiply injured patients. Br J Oral Maxillofac Surg 1996;34:303-8.

2. Hogg NJ, Stewart TC, Armstrong JE, Girotti MJ. Epidemiology of maxillofacial injuries at trauma hospitals in Ontario, Canada, between 1992 and 1997. J Trauma 2000;49:425-32.

3. Kassel EE, Noyek AM, Cooper PW. CT in facial trauma. J Otolaryngol 1983;12:2-15.

4. Leidner B, Beckman MO. Standardized whole-body computed tomography as a screening tool in blunt multitrauma patients. Emerg Radiol 2001;8:20-8.

5. Whitesell RT, Steenburg SD, Shen C, Lin H. Facial Fracture in the Setting of Whole-Body CT for Trauma: Incidence and Clinical Predictors. AJR Am J Roentgenol 2015;205:W4-10.

6. Bulla S, Blanke P, Hassepass F, Krauss T, Winterer JT, Breunig C, Langer M, Pache G. Reducing the radiation dose for low-dose CT of the paranasal sinuses using iterative reconstruction: feasibility and image quality. Eur J Radiol 2012;81:2246-50.

7. Hein E, Rogalla P, Klingebiel R, Hamm B. Low-dose $\mathrm{CT}$ of the paranasal sinuses with eye lens protection: effect on image quality and radiation dose. Eur Radiol 2002;12:1693-6.

8. Brem MH, Zamani AA, Riva R, Zou KH, Rumboldt Z, Hennig FF, Kikinis R, Norbash AM, Schoepf UJ. Multidetector CT of the paranasal sinus: potential for radiation dose reduction. Radiology 2007;243:847-52.

9. Bodelle B, Wichmann JL, Klotz N, Lehnert T, Vogl TJ, Luboldt W, Schulz B. Seventy kilovolt ultra-low dose CT of the paranasal sinus: first clinical results. Clin Radiol 2015;70:711-5.

10. Lu X, Lu Z, Yin J, Gao Y, Chen X, Guo Q. Effects of radiation dose levels and spectral iterative reconstruction levels on the accuracy of iodine quantification and virtual monochromatic CT numbers in dual-layer spectral detector CT: an iodine phantom study. Quant Imaging Med Surg 2019;9:188-200. 
11. Haubenreisser H, Meyer M, Sudarski S, Allmendinger T, Schoenberg SO, Henzler T. Unenhanced third-generation dual-source chest CT using a tin filter for spectral shaping at $100 \mathrm{kVp}$. Eur J Radiol 2015;84:1608-13.

12. Lell MM, May MS, Brand M, Eller A, Buder T, Hofmann E, Uder M, Wuest W. Imaging the Parasinus Region with a Third-Generation Dual-Source CT and the Effect of Tin Filtration on Image Quality and Radiation Dose. AJNR Am J Neuroradiol 2015;36:1225-30.

13. Wuest W, May M, Saake M, Brand M, Uder M, Lell M. Low-Dose CT of the Paranasal Sinuses: Minimizing $\mathrm{X}$-Ray Exposure with Spectral Shaping. Eur Radiol 2016;26:4155-61.

14. May MS, Brand M, Lell MM, Sedlmair M, Allmendinger T, Uder M, Wuest W. Radiation dose reduction in parasinus CT by spectral shaping. Neuroradiology 2017;59:169-76.

15. Petritsch B, Kosmala A, Weng AM, Bley TA. Tin-filtered $100 \mathrm{kV}$ ultra-low-dose CT of the paranasal sinus: Initial clinical results. PLoS One 2019;14:e0216295.

16. Deak PD, Smal Y, Kalender WA. Multisection CT protocols: sex- and age-specific conversion factors used to determine effective dose from dose-length product. Radiology 2010;257:158-66.

17. Landis JR, Koch GG. The measurement of observer agreement for categorical data. Biometrics 1977;33:159-74.

18. Primak AN, Ramirez Giraldo JC, Liu X, Yu L, McCollough CH. Improved dual-energy material discrimination for dual-source CT by means of additional spectral filtration. Med Phys 2009;36:1359-69.

19. Leyendecker P, Faucher V, Labani A, Noblet V, Lefebvre F, Magotteaux P, Ohana M, Roy C. Prospective evaluation of ultra-low-dose contrast-enhanced $100-\mathrm{kV}$ abdominal computed tomography with tin filter: effect on radiation dose reduction and image quality with a third-generation dual-source CT system. Eur Radiol 2019;29:2107-16.

20. Weis M, Henzler T, Nance JW Jr, Haubenreisser H, Meyer M, Sudarski S, Schoenberg SO, Neff KW,

Cite this article as: Ha JY, Baek HJ, Ryu KH, Cho E. Feasibility study of ultra-low-dose dedicated maxillofacial computed tomography using filter-based spectral shaping in patients with craniofacial trauma: assessment of image quality and radiation dose. Quant Imaging Med Surg 2021;11(4):12921302. doi: $10.21037 /$ qims-20-800
Hagelstein C. Radiation Dose Comparison Between $70 \mathrm{kVp}$ and $100 \mathrm{kVp}$ With Spectral Beam Shaping for Non-Contrast-Enhanced Pediatric Chest Computed Tomography: A Prospective Randomized Controlled Study. Invest Radiol 2017;52:155-62.

21. Braun FM, Johnson TR, Sommer WH, Thierfelder KM, Meinel FG. Chest CT using spectral filtration: radiation dose, image quality, and spectrum of clinical utility. Eur Radiol 2015;25:1598-606.

22. Mozaffary A, Trabzonlu TA, Kim D, Yaghmai V. Comparison of Tin Filter-Based Spectral Shaping CT and Low-Dose Protocol for Detection of Urinary Calculi. AJR Am J Roentgenol 2019;212:808-14.

23. Dewes P, Frellesen C, Scholtz JE, Fischer S, Vogl TJ, Bauer RW, Schulz B. Low-dose abdominal computed tomography for detection of urinary stone disease Impact of additional spectral shaping of the X-ray beam on image quality and dose parameters. Eur J Radiol 2016;85:1058-62.

24. Neisius A, Thomas C, Roos FC, Jager W, Tsaur I, Bartsch G, Knoll T, Haferkamp A. Urolithiasis 2016: Reliable, effective and low radiation exposure. Urologe A 2016;5 5:1291-6.

25. Wichmann JL, Hardie AD, Schoepf UJ, Felmly LM, Perry JD, Varga-Szemes A, Mangold S, Caruso D, Canstein C, Vogl TJ, De Cecco CN. Single- and dual-energy CT of the abdomen: comparison of radiation dose and image quality of 2 nd and $3 \mathrm{rd}$ generation dual-source CT. Eur Radiol 2017;27:642-50.

26. Bodelle B, Fischbach C, Booz C, Yel I, Frellesen C, Kaup M, Beeres M, Vogl TJ, Scholtz JE. Single-energy pediatric chest computed tomography with spectral filtration at 100 $\mathrm{kVp}$ : effects on radiation parameters and image quality. Pediatr Radiol 2017;47:831-7.

27. Kim CR, Jeon JY. Radiation dose and image conspicuity comparison between conventional $120 \mathrm{kVp}$ and $150 \mathrm{kVp}$ with spectral beam shaping for temporal bone CT. Eur J Radiol 2018;102:68-73. 